\title{
Investigation of O-Band Brillouin Fiber Laser Flatness at Different SOA Location
}

\author{
H. Ahmad, ${ }^{*}$ S. F. Norizan, M.Z. Zulkifli, and C. H. Pua \\ Photonics Laboratory, Department of Physics, University of Malaya, \\ 50603 Kuala Lumpur, Malaysia Tel: 603-79674290, Fax: 603-79676770 \\ Z.A.Ghani \\ Faculty of Applied Sciences, MARA University of Technology, 40450 Shah Alam, Selangor, Malaysia \\ S. W. Harun \\ Department of Electrical Engineering, Faculty of Engineering, \\ University of Malaya, 50603 Kuala Lumpur, Malaysia
}

\begin{abstract}
In this paper, we present the different amplitude flatness of O-band Brillouin fiber laser resulting from placing the $\mathrm{O}$ band semiconductor optical amplifier at different locations. Stable and flatter peaks of multiwavelength Brillouin were generated by placing the SOA after the nonlinear medium instead of placing it before the nonlinear medium. The maximum number of Stokes are also differs between both configuration where the maximum number of stokes achieved was 12 generated by $1320 \mathrm{~nm}$ Brillouin pump with spacing channels of $12.72 \mathrm{GHz}$.
\end{abstract}

KEYWORDS: O band semiconductor optical amplifier, multiwavelength Brillouin fiber laser

\section{INTRODUCTION}

Multiwavelength Brillouin fiber laser (BFL) has generated great interests due to its unique properties which include narrow linewidth, low threshold powers and also its simplicity. It has been of significant important in a number of applications including Brillouin sensors [1], frequency converters [2], slowing down of the velocity of light [3], and also dense wavelength division multiplexing (DWDM) system [4]. Many different methods have been demonstrated to generate multiwavelength BFL utilizing various amplification media including Erbium doped fiber (EDF) [5], Raman amplifier [6] and Ytterbium doped fiber [7]. The amplification media determines the operating region as well as optical characteristic of the laser such as optical signal noise ratio (OSNR), flatness of the output power, output power level, stable lasing output and broad wavelength tunability [8]. For that reason, it is important to investigate the effect of location of amplifier in the Brillouin fiber laser setup.

In this paper, a multi-wavelength BFL is demonstrated to operate in O-band region using a piece of TrueWave fiber (TWF) and a semiconductor optical amplifier (SOA) as gain media. The effect of the SOA location is also investigated to produce flat peak power of the multi-wavelength output. The flatness of the output peaks powers is important especially in DWDM applications as well as device testing applications.

*E-MAIL: harith@um. edu.my

\section{EXPERIMENTAL SETUP}

Figure 1 illustrates two linear cavity of Brillouin SOA fiber laser (BSFL). Both of the configurations are the same except for the location of SOA. Both configurations consist of an external cavity tunable laser source as a Brillouin Pump (BP), $20 \mathrm{~km}$ long TWF as Brillouin gain medium with mode field diameter of less than $8.4 \mu \mathrm{m}$ and an attenuation coefficient of $0.34 \mathrm{~dB} / \mathrm{km}$ at an operation wavelength of $1310 \mathrm{~m}$, O-band semiconductor optical amplifier (SOA) to amplify the BP and Stokes that produced from the cavity, a polarization controller to change the polarization of BP, and two mirrors like reflectors at both end of the linear cavity.

The two mirrors are constructed using 3 ports optical circulators and $1 \times 2$ couplers. For mirror 1, optical circulator CIR1 is connected to coupler $\mathrm{C} 1$ with a coupling ratio of 70:30. The reflection is created by connecting port 3 and port 1 of CIR 1 with $30 \%$ leg and common leg of $\mathrm{C} 1$ respectively. As a result, any signal coming from port 2 that transmitted to port 3 will flow through $\mathrm{C} 1$ with transmission of $30 \%$ of its power and entered port 1 of $\mathrm{OC} 1$ to be transmitted back at port 2 . This arrangement will created mirror with $30 \%$ reflectivity. Meanwhile, the $70 \%$ leg is connected to BP to channel the higher pump power into the resonator to overcome the Brillouin threshold. For mirror 2, the optical circulator CIR2 was connected to coupler $\mathrm{C} 2$ with coupling ration of 90:10. Port 3 of CIR2 was connected to common leg of C2 while its port 1 was connected to $90 \%$ leg of C2. Signal that approached from port 2 CIR2 will appear at port 3 which then run through common legs of $\mathrm{C} 2$ and split its power. $90 \%$ of its power were transmitted back to port 2 CIR 2 through its port 1, while another $10 \%$ of the signal power used to analyzed by connecting 


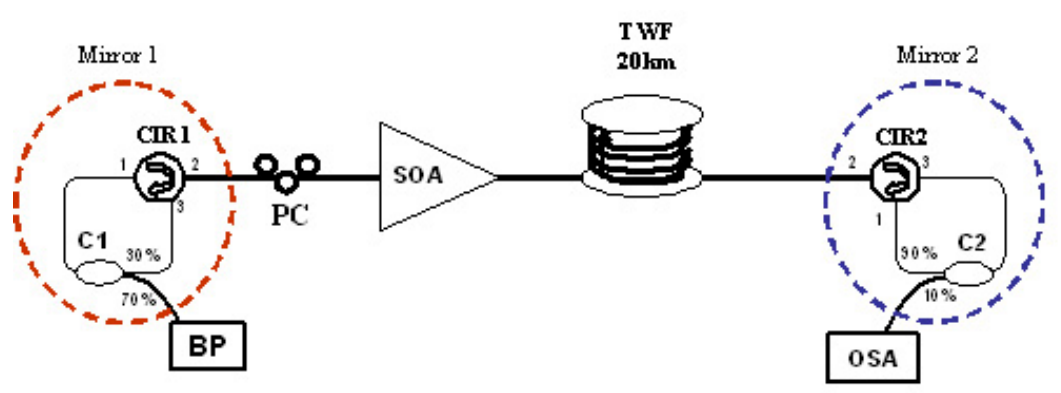

(a)configuration A

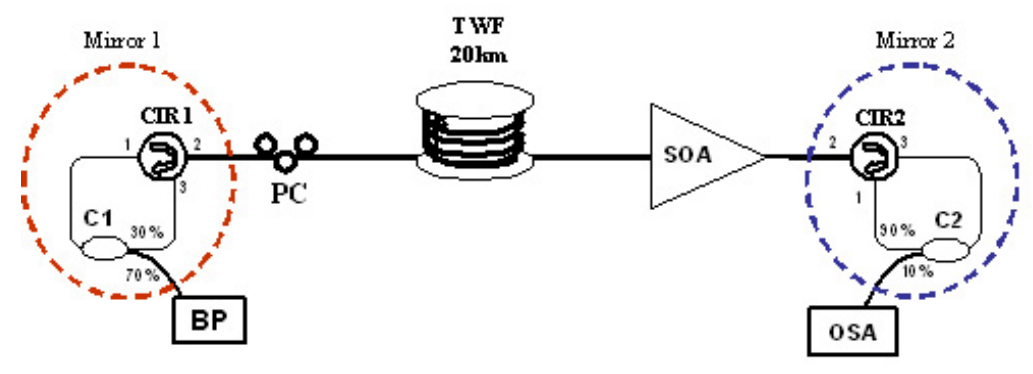

(b)configuration B

FIG. 1: Experimental set up of linear cavity of Brillouin SOA Fiber Laser for different SOA location

it to optical spectrum analyzer (OSA).

For configuration A of Figure 1(a), 70\% of BP power was transmitted from $\mathrm{C} 1$ through port 1 of CIR1 which subsequently entered its port 2. The SOA then amplified the BP power where the gain characteristic is as shown in Figure 2. The amplified BP was then interacts with the nonlinear gain medium TWF to generate the 1st Stokes which moving at the opposite direction of BP.

The $1^{\text {st }}$ Stokes that generated, were then amplified by the SOA and moving towards mirror 1 , which then reflected only $30 \%$ of its power back to the SOA to be amplified again. The amplified 1st Stokes now interacts with TWF as BP to generate 2nd Stokes, this process will continue take place until the latest Stokes have no sufficient power to be BP for the following Stokes. At the same time, the peak power of the Stokes will amplify by the Brillouin amplifier and until its reach its saturated gain. Meanwhile, the remaining BP that past through TWF was reflected by mirror 2 and back to TWF, which will generate Stokes if it has sufficient power.

As for configuration $\mathrm{B}, 70 \%$ power of $\mathrm{BP}$ from $\mathrm{C} 1$ emitted at port 2 CIR1 which was connected directly to the TWF. The BP then interacts with TWF to produce 1st Stokes moving opposite direction of BP. The Stokes then reflected back to the TWF by mirror 1 with reflectivity of $30 \%$. As for survived transmitted $\mathrm{BP}$, it was amplified by the SOA at the end of TWF, where the BP then reflected by mirror 2 with reflectivity of $90 \%$ and BP again be reamplified by the SOA. The amplified BP will then again interact with TWF to generated $1^{\text {st }}$ Stokes which move towards the SOA to be amplified and reflected by mirror 2 . The reflected 1 st Stokes then again amplified by SOA before entering the TWF as BP for $2^{\text {nd }}$ Stokes. This process continues until the latest Stokes has less power to generated new Stokes.

\section{RESULTS AND DISCUSSION}

Figure 2 shows the important gain characteristic of SOA used in this experiment. Figure 2(a) shows the gain of SOA at different wavelength, with small input power of $-30 \mathrm{dBm}$, the maximum gain of $16 \mathrm{dBm}$ obtain at wavelength $1315 \mathrm{~nm}$. Whereas for figure 2(b), the graph shows signal input power against its gain using SOA, which give information of saturation power. From the graph it shows that gain of SOA is decreased as the input power increased. The saturation input power of the SOA is defined as a point at which the gain reduced half $(3 \mathrm{~dB})$ from its maximum value. The saturation power is estimated to be approximately $-8 \mathrm{dBm}$.

Figure 3 shows the experimental results for both BFLs. Figures 3(a) and 3(b) illustrates the output spectrum with configuration $\mathrm{A}$ and $\mathrm{B}$, respectively. For configuration $\mathrm{A}$, the total Stokes generated is 11 , where the highest peak power is -19.64 $\mathrm{dBm}$. Unfortunately the Stokes generated from configuration A have uneven peak Stokes where the peak power different between all 11 Stokes is greater than $3 \mathrm{~dB}$ which show that this BFL is unsuitable to be used in most of applications that required its output power to be flat. With configuration $B$, the number of Stokes generated are 8 which is less compared to configuration A with the highest peak is observed to be -22.61 $\mathrm{dBm}$ as shown in Figure 3(b). However, the first 4 Stokes generated have flatter output which is less than $3 \mathrm{~dB}$ different of peak power compared to configuration $\mathrm{A}$. This BFL is suitable for many applications due to it's flatten properties.

These results show that the position of SOA plays an im- 


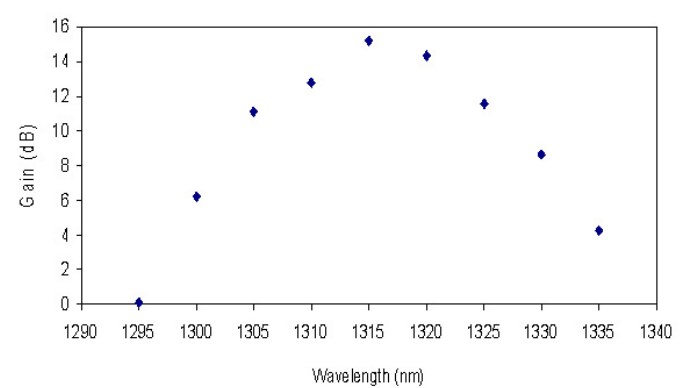

(a)

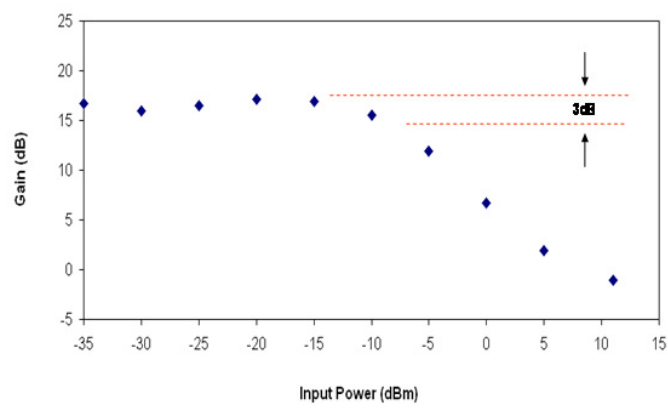

(b)

FIG. 2: Characteristics of 1310nm SOA (a) gain spectrum (b) gain characteristic against input signal powers.

portant role in the linear cavity multi-wavelength BFL. In configuration A, the SOA is placed between the BP and the nonlinear medium (TWF), which results in the BP intensity being amplified first by the SOA before penetrating into the TWF. It is known that having an input power of more than -8 $\mathrm{dBm}$ the SOA will experience less gain as it shown in Figure 2(b), hence in the case of Brillouin stokes generation, the BP power will reduce and affect the generation of Brillouin Stokes. Furthermore, in configuration A, due to the low reflection from mirror makes the Stokes arduous to achieve its saturation power after amplified by SOA, where resulted as uneven peak power of Stokes. For configuration B, high reflection of mirror 2 causes part of the S5tokes reach its saturation point and produce even peaks power.

Figure 4 shows the peak power of the transmitted Brillouin pump and the first 3 Stokes at different BP power for both configurations. It appears that, for configuration A the cavity have inadequate power to generate the $3^{\text {rd }}$ Stokes. The peak powers for transmitted Brillouin pump and the other 2 Stokes are not equal to each other as compared to configuration B, where the peak powers for BP and its first 3 Stokes are nearly the same at $-22 \mathrm{dBm}$.

Figure 5 shows the different number of stokes at different wavelengths for both configuration $\mathrm{A}$ and configuration B. The maximum number of Stokes created for configuration $\mathrm{A}$ is 10 , at wavelength $1310 \mathrm{~nm}$, while there are 12 Stokes for configuration B at wavelength $1320 \mathrm{~nm}$.

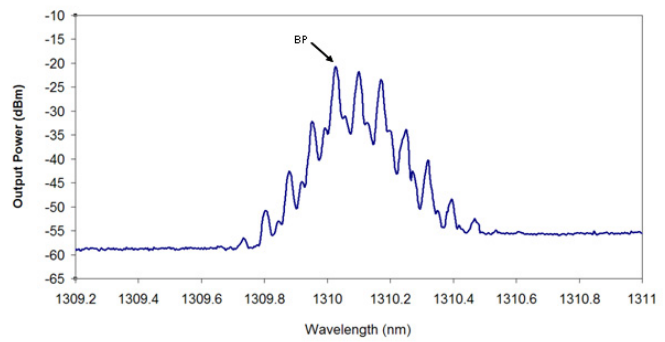

(a)

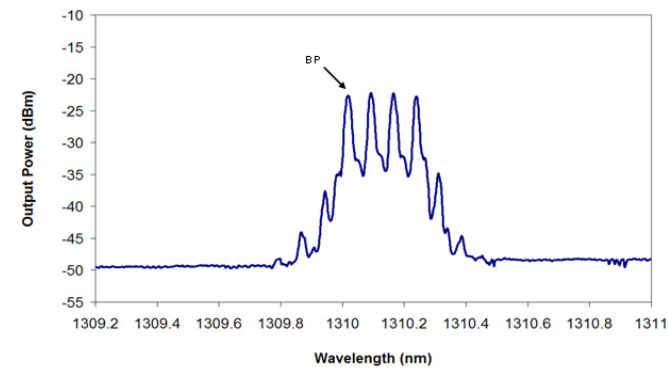

(b)

FIG. 3: Spectrum of Brillouin SOA fiber laser (BSFL) for (a) configuration $\mathrm{A}$ and (b) configuration $\mathrm{B}$.

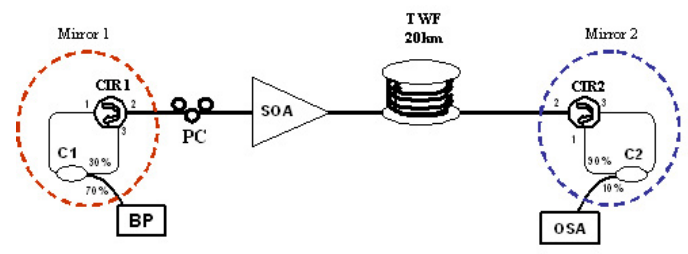

(a)configuration $\mathrm{A}$

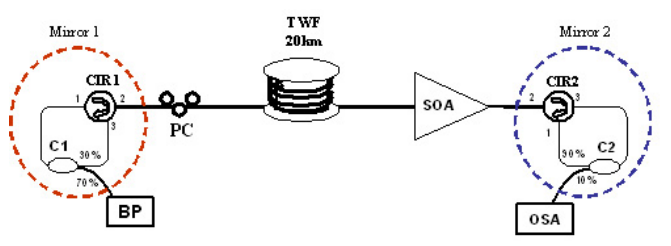

(b)configuration B

FIG. 4: Peak powers of transmitted Brillouin and the first three Stokes (a) for configuration A, (b) for configuration B.

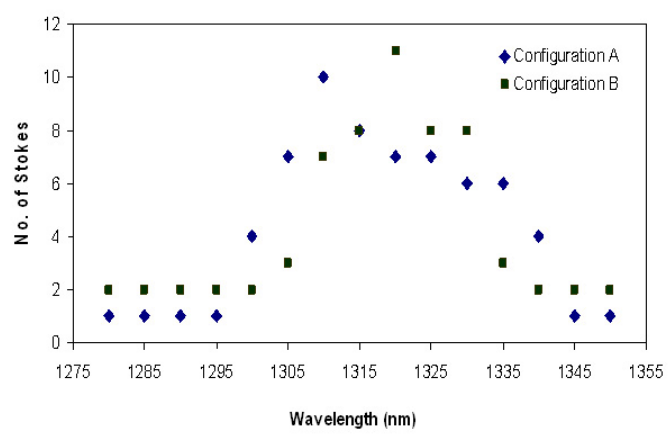

FIG. 5: Number of Stokes generated in configurations A and B. 


\section{CONCLUSION}

A multiwavelength BFL is demonstrated using a piece of TWF and SOA to operate in O-band region. The output spectrum is observed to be significantly changed with the location of SOA in the linear cavity. The peak powers of the Brillouin
Stokes are observed to be flatter with peak power variation of less than $3 \mathrm{~dB}$ difference using configuration $\mathrm{B}$, which was obtained by placing the SOA after the nonlinear medium. The maximum number of Stokes of 10 lines and 20 lines are obtained with configuration $\mathrm{A}$ and $\mathrm{B}$ at wavelength region of $1310 \mathrm{~nm}$ and $1320 \mathrm{~nm}$, respectively.
[1] M. A. Soto, G. Bolognini and F. D. Pasquale, IEEE Photonics Technology Letters, 21(7), pp. 450-452 (2009).

[2] Y. Shen, X. Zhang, G. Shen, K. Chen, Optics Communications 249, pp. 109-115 (2005).

[3] M. Gonzlez-Herrez, K.Y. Song, and L. Thvenaz, Applied Physics Letters 87, pp. 081113, (2005).

[4] S. Shahi, S. W. Harun, K. Dimyati, M. R. Tamjis and H. Ahmad,Microwave and Opt. Technol. Lett. 52, No. 6, pp. 1416$1418,(2010)$.

[5] M. H. Al-Mansoori, and M. A. Mahdi,Optic Express 16, pp.
7649-7654 (2008)

[6] A. K, Zamzuri, M.A. Mahdi, A. Ahmad, M. I. Md Ali, and M. H. Al-Mansoori, Optics Express, 15 (6), pp. 3000-3005 (2007)

[7] L. T. S. Ting, L. T. Fat and S. W. Harun, Brillouin Erbium Ytterbium Fiber Laser, Proceeding of the International Conf. on Computer and Communication Engeneering 2008, Kuala Lumpur, Malaysia, pp. 143-147, 2008

[8] D. Liu, N. Q. Ngo, X. Y. Dong, S. C. Tjin, and P. Shum, Applied Physic B, 81, pp. 807-811(2005) 